\title{
Analysis of Different Types of Regret in Continuous Noisy Optimization
}

\author{
Sandra Astete-Morales, Marie-Liesse Cauwet, Olivier Teytaud \\ TAO/Inria Saclay-IDF, Univ. Paris-Saclay \\ Bat. 660, rue Noetzlin, Gif-Sur-Yvette, France
}

October 10, 2018

\begin{abstract}
The performance measure of an algorithm is a crucial part of its analysis. The performance can be determined by the study on the convergence rate of the algorithm in question. It is necessary to study some (hopefully convergent) sequence that will measure how "good" is the approximated optimum compared to the real optimum.

The concept of Regret is widely used in the bandit literature for assessing the performance of an algorithm. The same concept is also used in the framework of optimization algorithms, sometimes under other names or without a specific name. And the numerical evaluation of convergence rate of noisy algorithms often involves approximations of regrets. We discuss here two types of approximations of Simple Regret used in practice for the evaluation of algorithms for noisy optimization. We use specific algorithms of different nature and the noisy sphere function to show the following results. The approximation of Simple Regret, termed here Approximate Simple Regret, used in some optimization testbeds, fails to estimate the Simple Regret convergence rate. We also discuss a recent new approximation of Simple Regret, that we term Robust Simple Regret, and show its advantages and disadvantages.
\end{abstract}

\section{Introduction}

The performance measure of an algorithm involves the evaluation of the quality of the approximated optimum with regards to the real optimum. This can be done by using the concept of Regret, well studied in the machine learning literature and used in the noisy optimization literature, sometimes under other names. Basically, in the optimization framework, the regret acounts for the "loss" of choosing the point used in the algorithm over the best possible choice: the optimum. Therefore, we measure the difference between the point used/recommended by the algorithm and the optimum in terms of the objective function.

In general, an optimization algorithm searches for the optimum, and to do so, it produces iteratively search points which will be evaluated through the objective function. And at regular steps, the algorithm must return a recommendation point that will be the best approximation to the optimum so far. 
Note that the recommendation point can be equal to a search point, but not necessarily.

The most usual form of regret is termed Simple Regret. The Simple Regret measures the distance, in terms of fitness values, between the optimum and the recommendation point output by the algorithm. It is widely used (possibly without this name) in noisy optimization $18,14,12$. However, some test beds, notably the Bbob/Coco framework in the first version, did not allow the distinction between search points (at which the fitness function is evaluated) and recommendations (which are output by the algorithm as an approximation of the optimum), so that the Simple Regret can not be checked. This leads to the use of an Approximate Simple Regret (name by us), which evaluates the fitness difference between the search points (and not the recommendations) and the optimum. Later, another form of regret, that we will term here Robust Simple Regret, was also proposed, using recommendation points. We analyze in this paper the use of different regrets that aim to estimate the quality of the approximated optimum in a similar way. In particular, we show to which extent they lead to incompatible performance evaluations of the same algorithm over the same class of noisy optimization problems, i.e. the convergence rate for the Approximate or the Robust Simple Regret overestimates or underestimates the convergence rate of the more natural simple regret. We also prove some new results in terms of Simple Regret itself.

\section{Framework and Regrets}

This section is devoted to the formalization of the noisy optimization problem considered and the analysed regrets. We will focus on the Simple Regret and the alternative definitions that aim to approximate it (denoted here Approximate Simple Regret and Robust Simple Regret). At the end of the section we will highlight some general relationships between the presented regrets.

\subsection{Continuous Noisy Optimization}

Given a fitness function $F: D \subset \mathbb{R}^{d} \rightarrow \mathbb{R}$, also known as objective function, optimization (minimization) is the search for the optimum point $x^{*}$ such that $\forall x \in D, F\left(x^{*}\right) \leq F(x)$. The fitness function is corrupted by additive noise. In other words, given a search point $x \in D$, evaluating $F$ in $x$ results in an altered fitness value $f(x, w)$ as follows:

$$
f(x, w)=F(x)+w,
$$

where $w$ is an independent random variable of mean zero and variance $\sigma$. In the present paper, we will consider a simple case, namely a standard Gaussian additive noise 1. In addition, we assume that $F(x)=\left\|x-x^{*}\right\|^{2}$, where $x^{*}$ is randomly uniformly drawn in the domain $D$. Noisy optimization is then the search for the optimum $x^{*}$ such that $\mathbb{E}_{w} f\left(x^{*}, w\right)$ is approximately minimum, where $\mathbb{E}_{w}$ denotes the expectation over the noise $w$.

Consider a noisy black-box optimization scenario: for a point $x$, the only available information is the noisy value of $F$ in $x$ as given by $f(x, w)$ for some

\footnotetext{
${ }^{1}$ More general cases such that $\mathbb{E} f(x, w)=F(x)$ can be considered, as most algorithms do not request the noise to be additive and independent; the key point is the absence of bias.
} 
independent $w$. An optimization algorithm generates $x_{1}, x_{2}, \ldots, x_{n}, \ldots$, successive search points at which the objective function is evaluated in a noisy manner. It also generates $\tilde{x}_{1}, \tilde{x}_{2}, \ldots, \tilde{x}_{n}, \ldots$ which are recommendations or approximations of the optimum after $n$ fitness evaluations are performed.

\subsection{Simple Regret and variants}

The Simple Regret $(S R)$ focuses only on approximating the optimum in terms of fitness values. Its definition is:

$$
S R_{n}=\mathbb{E}_{w}\left(f\left(\tilde{x}_{n}, w\right)-f\left(x^{*}, w\right)\right)=F\left(\tilde{x}_{n}\right)-F\left(x^{*}\right)
$$

Notice that the expectation operates only on $w$ in $f\left(\tilde{x}_{n}, w\right)$, and not on $\tilde{x}_{n}$. As a consequence $S R_{n}$ is a random variable due to the stochasticity of the noisy evaluations of the search points or the (possible) internal randomization of the optimization algorithm.

The $S R$ can be a part of the performance evaluation of an algorithm. In the noise-free case it can be used to determine the precision of a method, by ensuring that the algorithm outputs a recommendation $\tilde{x}_{m}$ satisfying $S R_{m} \leq \epsilon$. Even more, when testing algorithms, it is common to use the "first hitting time" (FHT). FHT in fact refers to the first "stable" hitting time, i.e. the next recommendation is at least as good as the previous one. This is a reasonable assumption for algorithms solving noise-free problems. In this case the FHT is the minimum $n$ such that $S R_{n} \leq \epsilon$, provided that the recommendation is defined as $\tilde{x}_{n}=x_{i(n)}$ with $1 \leq i(n) \leq n$ minimizing $S R_{i(n)}$. However, there is no exact equivalence or natural extension for the FHT with precision $\epsilon$ on noisy optimization. The algorithm only has access to noisy evaluations hence it cannot compute with "certainty" $S R_{n}$, which corresponds to the precision of the algorithm.

An alternative definition, that aims to measure the precision in a similar way to $S R$, is the Approximate Simple Regret2 $(A S R)$, defined by:

$$
A S R_{n}=\min _{m \leq n} F\left(x_{m}\right)-F\left(x^{*}\right) .
$$

$A S R$ takes in account the "best" evaluations among all the search points. It is used in the Bbob/Coco framework [7, 8, 19, 9, 26, 27, 23, 31, 22, and in some theoretical papers [15]. Notice that since $A S R$ is non-increasing, the FHT can be computed.

In this paper we will also discuss another variant of regret, the Robust Simple Regret3 $(R S R)$, defined by:

$$
R S R_{n}=\min _{k \leq n} \max _{(k-\lfloor g(k)\rfloor)<m \leq k}\left(F\left(\tilde{x}_{m}\right)-F\left(x^{*}\right)\right),
$$

where $g(n)$ is a polylogarithmic function of $n$ and $\lfloor\cdot\rfloor$ is the floor function. The $R S R$ is the "best" SR since the beginning of the run, sustained during $\lfloor g(k)\rfloor$ consecutive recommendations 4 . The polylogarithmic nature of $g(\cdot)$ is

\footnotetext{
${ }^{2}$ The name is proposed by us.

${ }^{3}$ Discussed on Bbob-discuss mailing list (http://lists.lri.fr/pipermail/bbob-discuss/2014-0ctober.txt The name is proposed by us.

${ }^{4}$ If $(k-\lfloor g(k)\rfloor)<0$, then the max on the definition considers indexes between 1 and $k$
} 
explained by the following argument: $g(k)$ be large enough, so that we have a correct recommendation confirmed over $g(k)$ iterations, but small enough, so that we do not have to wait many evaluations before acknowledging that a correct recommendation has been found. The $R S R$ uses the recommendation $\tilde{x}_{m}$ instead of the search point $x_{m}$ used in $A S R$. But it uses the worst of a sequence of recommendations.

As a side note about the definition of $R S R$, it was originally proposed to use a quantile instead of the maximum. The "quantile version" (without this name), was proposed to become part of the performance measure in Bbob/Coco. However, we will show that it is possible to get a $R S R$ decreasing quicker than the $S R$, so that $R S R$ is a poor approximation of $S R$. The result is valid even with the quantile $100 \%$, i.e. the maximum. The same is possible with any other quantile.

The introduction of $R S R$ apparently outplays $A S R$ as an approximation of $S R$ by two means. First, by using recommendations rather than search points. Second, by checking on multiple recommendations that the optimum is correctly found with a given precision. In addition, as well as $A S R$, it is nonincreasing, therefore it can be used for fastening experiments on testbed. Please note however that this advantage makes sense only when the target fitness value is known, which is rarely the case except in an artificial testbed.

To investigate the convergence rate of the regrets, we will use a slightly different notation than classical works on noisy optimization. Usually the rates are given in terms of $O(h(n))$ where $h(n)$ is some function depending on the number of evaluations $n$. The state of the art shows that in many cases $([17$, [18, 29, 15]) $S R_{n}=O\left(n^{\psi}\right)$ where $\psi<0$ implies that the algorithm converges. Therefore, there is a linear relationship between $\log \left(S R_{n}\right)$ and $\log (n)$ with a slope $\psi$, where $\log (\cdot)$ is the natural logarithm. We will then refer to the slope of the regret when speaking about the convergence rate of the regret. The definition of the slope of the $S R$ is:

$$
s(S R)=\limsup _{n \rightarrow \infty} \frac{\log \left(S R_{n}\right)}{\log (n)}
$$

We have the corresponding definition for the slope of $A S R$ and $R S R$. Notice that if the slope is close to 0 , then the algorithm (at best) converges slowly. On the contrary, if the slope is negative and far away from 0 , then the algorithm is fast.

\subsection{General results for $S R, A S R$ and $R S R$ : $R S R$ is an op- timistic approximation of $S R$}

The problems analysed in this paper arise from the gap between $s(S R)$ and $s(A S R)$ or $s(R S R)$. Ideally we would like to have a regret that can be used easily and that approximates the Simple Regret. In the following sections (3) and 4) we will see with specific examples there is indeed a gap between $s(S R)$ and $s(A S R)$. In some cases using $A S R$ overestimates the performance of algorithms and in others it underestimates their performance. An extreme case is detailed in section 4, where we prove that Alg. 3 has optimal convergence rate in term of $S R$ whilst for $A S R$ it does not converge at all.

In general, by definition, we have that for any algorithm, $s(R S R) \leq s(S R)$. From this point of view, $R S R$ is a correct lower bound for $S R$. In other words, 
if an algorithm is fast in terms of $S R$, its performance measured by $R S R$ will be at least as good. Unfortunately, this bound is not nearly tight. We will prove that a small modification on the algorithm induces $s(R S R) \leq s(A S R)$ whereas $s(S R)$ is the same - so that, for cases in which $s(A S R)<s(S R)$ (sometimes by far, as explained in later sections), we get $s(R S R)<s(S R)$ (sometimes by far).

Let $A$ be an optimization algorithm and its search points $\left(x_{i}\right)_{i \geq 1}$. Consider another algorithm denoted $A_{g}$ and its successive search points $\left(X_{i}\right)_{i \geq 1}$. The search points of $A_{g}$ are obtained by repeating $\lfloor g(n)\rfloor$ times, for any $n$, the search points $x_{n}$ of $A$. Hence, we get the assignment:

$$
\begin{array}{rllll}
X_{1}= & \ldots & = & X_{1+\lfloor g(1)\rfloor} & \leftarrow x_{1} \\
X_{2+\lfloor g(1)\rfloor}= & \ldots & = & X_{2+\lfloor g(1)\rfloor+\lfloor g(2)\rfloor} & \leftarrow x_{2} \\
& \vdots & & & \\
X_{n+\sum_{j=1}^{n-1}\lfloor g(j)\rfloor}= & \ldots & = & X_{n+\sum_{j=1}^{n}\lfloor g(j)\rfloor} & \leftarrow x_{n}
\end{array}
$$

Let the recommendation points of $A_{g}$ be defined by $\tilde{X}_{n}=X_{n}$ for any $n$. Therefore $A_{g}$ is a slightly modified version of $A$ since there is an additional polylogarithmic number of evaluation in $A_{g}$, assuming $g$ is polylogarithmic. The $R S R$ of $A_{g}$ (say $R S R_{A_{g}}$ ) converges approximately as fast as the $A S R$ of the original algorithm (say $A S R_{A}$ ). The extra polylogarithmic number of evaluations does not affect the linear convergence in $\log / \log$ scale. Hence, for any algorithm $A, s\left(R S R_{A_{g}}\right) \leq s\left(A S R_{A}\right)$.

The general relationships between the slopes of the $S R$ and its approximations are not conclusive since the bounds are not tight. We will show some gaps between the different approximations of $S R$ and the $S R$ itself. In the following sections we present five algorithms that will serve as clear examples to see the differences of using one or another regret as performance measures. We will focus in two types of algorithms: the first group, in Section 3. consists of Evolutionary Algorithms and Random Search and the second, in Section 4, of algorithms using approximations of the gradient of the objective function. For each class of algorithms, we exhibit convergence rate bounds on $s(S R), s(A S R)$ and $s(R S R)$. Section 5 displays some experimental works in order to confront theory, conjecture and practice.

\section{Evolutionary Algorithms}

On the group of Evolutionary Algorithms (EAs), we present Random Search (RS), Evolution Strategy (ES) and Evolution Strategy with resampling $(\mathrm{ES}+\mathrm{r})$. They all use comparisons between fitness values to optimize the function. 


\subsection{Random Search}

Random Search (Alg. 1) is the most basic of stochastic algorithms 24. The search points $x_{1}, \ldots, x_{n}, \ldots$ are independently identically drawn according to some probability distribution. $\tilde{x}_{n}$ is usually the search point with the best fitness so far, i.e. with $y_{i}$ the fitness value obtained for $x_{i}$, we have $\tilde{x}_{n}=x_{i}$ with $i \in\{1, \ldots, n\}$ minimizing $y_{i}$.

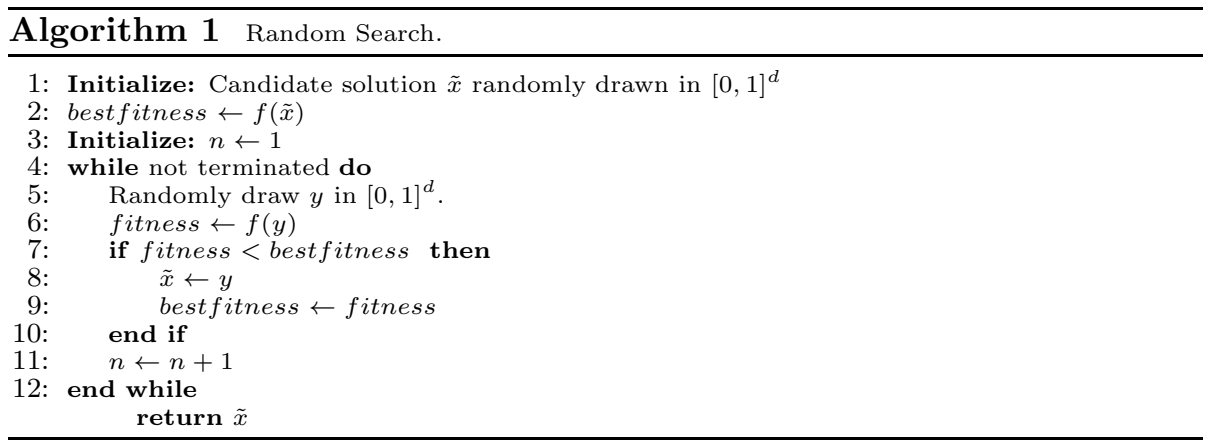

We consider in this paper a simple variant of RS to show clearly the contrast between $S R$ and $A S R$.

Framework for RS: each search point is randomly drawn independently and uniformly, sampled once and only once, with the uniform probability distribution over $[0,1]^{d}$. The objective function is the noisy sphere function $f$ :

$$
f(x)=\left\|x-x^{*}\right\|^{2}+\mathcal{N} .
$$

where $\mathcal{N}$ is a standard Gaussian variable.

In this setting, existing results in the literature imply a bound on $s(A S R)$ as explained in Property 1. We will prove then that the slope of the Simple Regret is not negative, as formalized in Theorem 1 .

\subsubsection{Approximate Simple Regret: $s(A S R)=-2 / d$}

Property 1. Consider RS described in Alg. 1, with the framework above. Then almost surely $A S R_{n}=O\left(\frac{1}{n^{2 / d}}\right)$.

Proof. [16] has shown that among $n$ points generated independently and uniformly over $[0,1]^{d}$ the closest search point to the optimum is almost surely at distance $O\left(\frac{1}{n^{1 / d}}\right)$ from the optimal point $x^{*}$ within a logarithmic factor. Hence for the sphere function 5 , the Approximate Simple Regret $A S R_{n}$ almost surely satisfies: $A S R_{n}=O\left(\frac{1}{n^{2 / d}}\right)$ up to logarithmic factors.

\subsubsection{Simple Regret: $s(S R)$ is not negative}

Theorem 1. With the framework above, for all $\beta>0$, the expected simple regret $\mathbb{E}\left(S R_{n}\right)$ is not $O\left(n^{-\beta}\right)$.

Proof. See supplementary material.

\footnotetext{
${ }^{5}$ The result also holds for a function locally quadratic around a unique global optimum.
} 
Remark: Roughly speaking, the proof of the theorem is based on the fact that with a non-zero probability a search point which does not have the best fitness value, is selected as the best point in Lines 7].9] of Alg. [1.

\subsection{Evolution Strategies $(E S)$}

Evolution Strategies [25, 28] are algorithms included in the category of Evolutionary Algorithms (EAs). In general, EAs evolve a population until they find an optimum for the objective or fitness function. The process starts by a population randomly generated. Then the algorithm iterates creating new individuals using crossover and mutation and then evaluating this new population of offspring and selecting the ones - regarding to their fitness values - that will become the parents of the next generation.

ES have some more specific selection and mutation processes. Usually the mutation is performed by creating new individuals starting from the parent and adding a random value to it (usually normally distributed around the parent). There are various rules for choosing the step-size.The selection in ES is usually deterministic and rank-based. This is, the individuals chosen to be the parents of the next generations are the ones that have the best fitness values.

When dealing with noisy function, the sorting step of the ES is disturbed by the noise and misranking might occur. To tackle this problem, Arnold and Beyer, in [1, 2] propose to increase the population size. An alternative is to evaluate multiple times the same search point and average the resulting fitness values. For a given search point $x \in D, r$ evaluations are performed: $\left(f\left(x, w_{1}\right), \ldots, f\left(x, w_{r}\right)\right)$ and the fitness value used in the comparisons is the average of these evaluations $\frac{1}{r} \sum_{i=1}^{r} f\left(x, w_{i}\right)$. In particular, the variance of the noise is divided by $r$. Several rules have been studied: constant 13, adaptive (polynomial in the inverse of the step-size), polynomial and exponential 5 . number of resamplings. A general $(\mu, \lambda)$-ES is presented in Algorithm 2

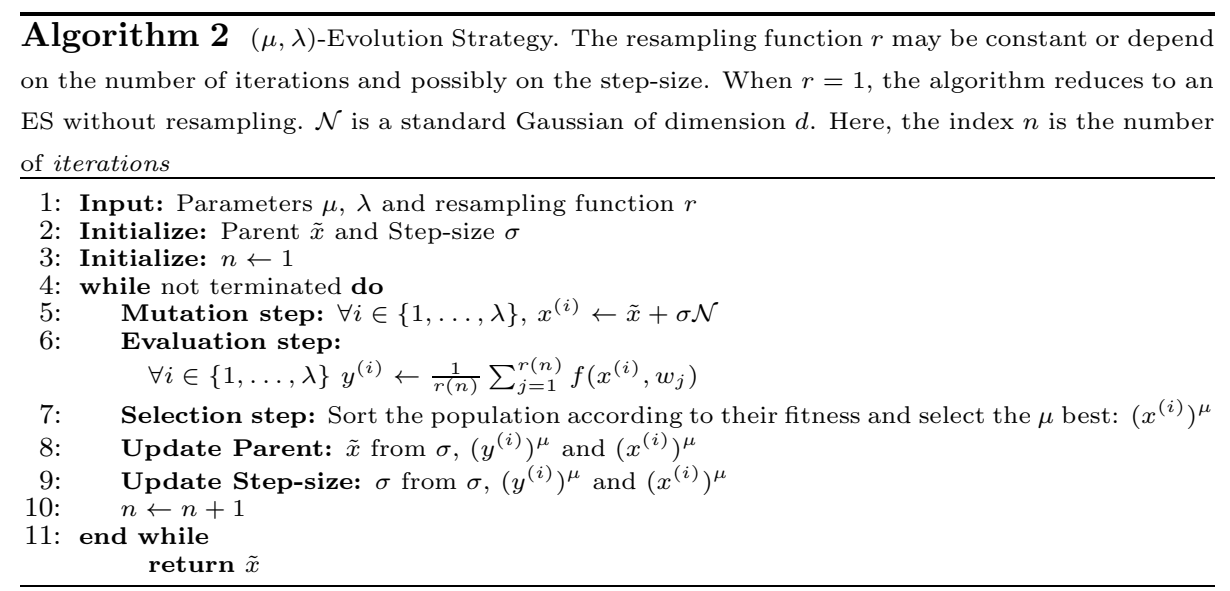

\subsubsection{Regrets for ES without resampling}

It is known [3] that when the noise strength is too big, classical evolution strategies (without reevaluations or other noise adaptation scheme) do not converge, they stagnate. [10] experimentally shows that an ES without any adaptation to 
the noisy setting stagnates around some step-size and at some distance of the optimum. The divergence results suggest that the ES in this case is only as a more sophisticated version of RS. The steps of the ES are more complicated, but not sufficiently adapted to handle the noise of the function. We propose then a Conjecture on the convergence rates for ES.

Conjecture 1 (Convergence rates for ES). Evolution Strategies without a noise handling procedure have the same convergence rates as Random Search for all regrets.

\subsubsection{Simple regret for ES with resamplings}

We will see that the results are more encouraging than in Section 3.2.1 when we consider an ES with some adaptation to mitigate the effect of the noise. We will assume that the function $r$ (number of revaluations per point) in Alg. 2 grows polynomially or exponentially with the number of iterations.

The work in [5] shows that ES that include an exponential number of revaluations converges with high probability to the optimum. The convergence rate is $s(S R)=K$ for some $K<0$ under assumptions about the convergence in ES in the noise-free case. Moreover 4 shows that ES, under general conditions, must exhibit $K>-\frac{1}{2}$. There is no formal proof of an upper bound that can theoretically ensure a value or a range for $s(S R)$. However, the experiments on [5] suggest that the $K=-\frac{1}{2}$ is reached for functions with a quadratic Taylor expansion and additive noise (as in Eq. 1). Hence we propose Conjecture 2

Conjecture $2(S R$ for $\mathrm{ES}+r)$. Consider $0<\delta<1$. For some resampling parameters (i.e. for some revaluation function $r$ ), Evolution Strategies with resampling (Alg. Q 2) satisfy $s(S R)=-1 / 2$ with probability $1-\delta$.

This conjecture applies to some ES with step-size scaling as the distance to the optimum, i.e. $\sigma_{n}$ used for generating the $n^{\text {th }}$ search point has the same magnitude as $\left\|\tilde{x}_{n}-x^{*}\right\|([25,[1])$. [10] has proposed variants of ES for quickening the convergence thanks to large mutations and small inheritance. Such an approach is not covered by the bound in [4] and it is for sure an interesting research direction - maybe it might reach slope $s(S R)=-1$.

\subsubsection{Approximate simple regret for ES with resamplings}

We have seen that an ES can reach a slope of $S R$ approximately $-\frac{1}{2}$, when using resamplings. However, $A S R$ can be better by slightly modifying the original ES, and therefore achieving a faster convergence rate than the real one represented by $s(S R)$ We consider an ES - called $M E S+R$ for Modified ES with Resampling. Let $r_{n}$ be exponential in the number of iterations: $r_{n}=R \cdot \zeta^{n}, R>0, \zeta>1$. $M E S+R$ is as in Alg. 2 with the following modifications. At iteration $n$ :

Generation: (Alg. 2, Line 5) Generate additional $r_{n}$ "fake" offspring $\left\{x^{(i) f}: 1 \leq i \leq r_{n}\right\}$, with the same probability distribution as the $\lambda$ offspring. They will be evaluated one time each, but they will not be taken into account for the selection. Note that this means that they are part of the sequence of points considered by $A S R$, but not by $S R$.

Evaluation: (Alg. 2, Line 6) Evaluate $r_{n}$ times each "true" offspring $\left\{x^{(i)}\right.$ : $1 \leq i \leq \lambda\}$ to obtain their corresponding fitness value $y^{(i)}$. Evaluate one time 
each "fake" offspring. Therefore, performing $(\lambda+1) r_{n}$ function evaluations in each iteration.

Then, under some reasonable convergence assumptions which are detailed in theorem 2 below, the $A S R$ reaches a faster rate: $s(A S R)=-1 / 2-2 / d$ with high probability.

Theorem 2. Consider $0<\delta<1$. Consider an objective function $F(x)=\|x\|^{2}$, where $x \in \mathbb{R}^{d}$. Consider a MES $+R$ as described previously. Assume that:

(i) $\sigma_{n}$ and $\left\|\tilde{x}_{n}\right\|$ have the same order of magnitude:

$$
\left\|\tilde{x}_{n}\right\|=\Theta\left(\sigma_{n}\right) .
$$

(ii) $\log -\log$ convergence occurs for the SR:

$$
\frac{\log \left(\left\|\tilde{x}_{n}\right\|\right)}{\log (n)} \underset{n \rightarrow+\infty}{\longrightarrow}-\frac{1}{2} \text { with probability } 1-\delta
$$

Then, with probability at least $1-\delta, s(A S R)=-1 / 2-2 / d$.

Proof. See supplementary material.

Remark 1. The assumption of $s(S R)=-1 / 2$ is based on the convergence of ES in the noise-free case and it is essential to prove Theorem 0 . This rate of convergence can be proved when the ES converges in the noise-free case (details on [5]). But the convergence of ES has not been formally proved, not even for the noise-free case. There is an important element given in [6], showing that $\frac{1}{n} \log \left\|x_{n}-x^{*}\right\|$ converges to some constant, but this constant is not proved negative. Furthermore, parameters ensuring convergence in the noisy case are unspecified in [5].

\section{Stochastic Gradient Descent}

For the group of Stochastic Gradient Descent Algorithms, we consider the ones presented by Shamir 29] and Fabian [18] which approximate the gradient of the objective function. We will denote them Shamir algorithm and Fabian algorithm respectively. Both of them approximate the gradient of the function using function evaluations by different methods, therefore they remain in the blackbox category. Fabian algorithm uses the average of redundant finite differences and Shamir algorithm a one point estimate gradient technique.

The convergence rates in terms of $S R$ are proved in [29] and [18. For Shamir it is shown that $s(S R)=-1$ in expectation for quadratic functions. Fabian ensures a rate $s(S R)=-1$ approximately and asymptotically only for limit values of parameters. However, it requires only smooth enough functions, so the class of functions is wider than the one considered in [29]. This rate $s(S R)=-1$ has been proved tight in [14. Hence, Shamir and Fabian algorithm are faster than ES's, which cannot do better than $s(S R)=-\frac{1}{2}$, at least under their usual form [4]. 


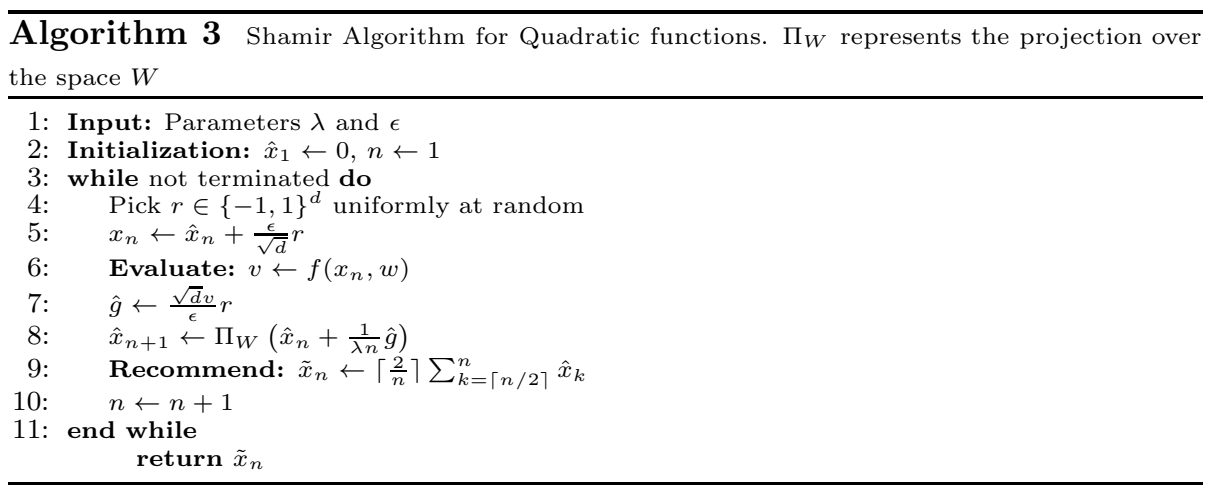

\subsection{Shamir's quadratic algorithm}

Shamir algorithm presented in [29] for quadratic functions is Algorithm 3 .

One of the key points in Alg. 3 are that there is only one evaluation per iteration (somehow in the spirit of Simultaneous Perturbation Stochastic Approximation SPSA 30, 20]). The second important point is that the expectation of the distance between search points and recommendations is constant, which implies that the search points do not converge towards the optimum! This is not a problem for the convergence in terms of $S R$, when search points $x_{n}$ and recommendations $\tilde{x}_{n}$ are distinguished, but it makes a difference for $A S R$.

Shamir algorithm has an optimal convergence rate in expectation $(s(S R)=$ $-1)$ for quadratic functions. This fact should be acknowledge by any other regret used to evaluate its performance which aims to aproximate the $S R$. But intuitively in the framework of Shamir algorithm, the $s(A S R)$ is presumably a bad approximation of $s(S R)$ due to the queries at a constant distance of the current recommendation. This convergence rate in terms of $s(A S R)$ could not be obtained from the results in 29 . Nonetheless, we prove in a general way that as long as the results for Shamir algorithm are satisfied almost surely, then $s(A S R)=0$ a.s. Therefore we present the latter result in Theorem 3 and a conjecture on the convergence rate of $s(A S R)$ in expectation for Shamir algorithm.

Theorem 3. Assume that the optimum $x^{*}$ is unique and that $\left(\tilde{x}_{n}\right)$ is a sequence of recommendation points converging a.s. to $x^{*}$. Assume that the sequence of evaluation points $\left(x_{n}\right)$ is such that $\forall n, x_{n} \neq x^{*}$ a.s. and that $\left\|x_{n}-\tilde{x}_{n}\right\|$ is constant. Then, a.s.

$$
s(A S R)=0 .
$$

Proof. $\tilde{x}_{n}$ converges almost surely to the optimum and $x_{n}$ is at a constant distance from $\tilde{x}_{n}$. Therefore the distance between $x_{n}$ and the optimum converges to a constant. This implies that the minimum $\min _{i=1}^{n}\left\|x_{i}-x^{*}\right\|^{2}$ is lower bounded by some constant. Therefore $s(A S R)=0$.

Conjecture 3 (ASR for the Shamir algorithm). Shamir algorithm also verifies $s(A S R)=0$ a.s. on quadratic functions. 


\subsection{Fabian Algorithm}

Algorithm 4 presents the algorithm studied in [18. Unlike Algorithm 3, Fabian algorithm performs several evaluations per iteration, and the distance between search point and recommandation decreases.

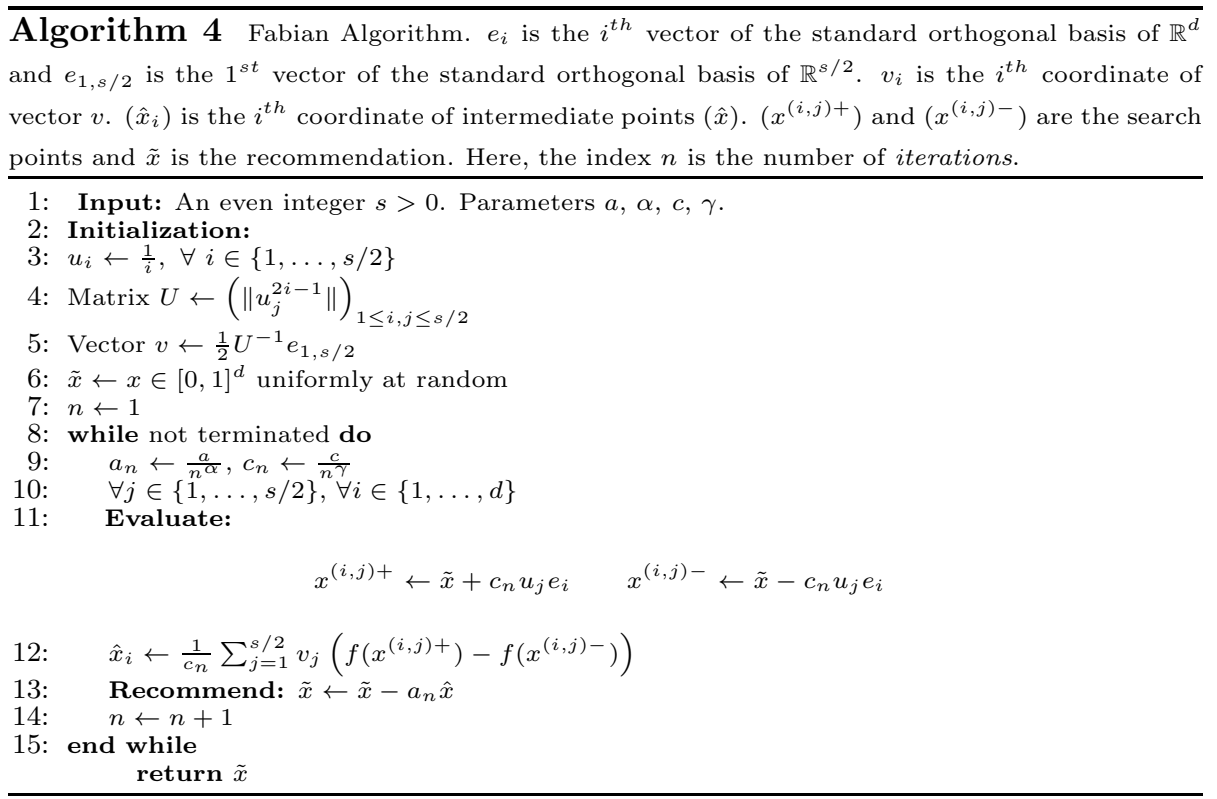

The work in 18 gives the convergence rate in terms of $S R$. The result is presented here as Theorem4. The value of the $s(S R)$ depends on the parameters of the algorithm and it is ensured a.s.

Theorem 4 (Simple Regret of Fabian algorithm). Let $s$ be an even positive integer and $F$ be a function $(s+1)$-times differentiable in the neighborhood of its optimum $x^{*}$. Assume that its Hessian and its $(s+1)^{\text {th }}$ derivative are bounded in norm. Assume that the parameters given in input of Algorithm 4 satisfy: $a>0, c>0, \alpha=1,0<\gamma<1 / 2$ and $2 \lambda_{0} a>\beta_{0}$ where $\lambda_{0}$ is the smallest eigenvalue of the Hessian. Let $\beta_{0}=\min (2 s \gamma, 1-2 \gamma)$. Then, a.s.:

$$
n^{\beta}\left(\tilde{x}_{n}-x^{*}\right) \rightarrow 0 \forall \beta<\beta_{0} / 2
$$

In particular, when $F$ is smooth enough, we get $s(S R)=-2 \beta$.

Remark 2. Note that $s(S R)$ optimal when $\gamma=\frac{1}{2}(s+1)^{-1}$. In this case, $\beta_{0}=\frac{s}{s+1} \underset{s \rightarrow \infty}{\rightarrow} 1$. $\beta_{0}$ can be made arbitrarily close to 1 , so $2 \beta$ also, but then $\gamma$ goes to 0 . Hence we get the values of Table 1, with $2 \beta=1-e, e>0$ and close to 0 .

This shows that the Fabian algorithm can have $s(S R)$ arbitrarily close to -1 , which is optimal. As in the case of Shamir, this optimal performance should be captured by the regret used to evaluate the algorithm. Unfortunately, this is not the case, as detailed in Theorem 5. 
Theorem 5 (ASR of Fabian algorithm). Let $F$ be a $\lambda$-convex and $\mu$-smooth function corrupted by an additive noise with upper bounded density and with optimum randomly drawn according to a distribution with upper bounded density. Then, a.s.,

$$
s(A S R)=-\min (2 \beta, 2 \gamma) .
$$

Proof. See supplementary material.

Remark 3. Theorem 5 shows that $s(A S R)=-\min (2 \beta, 2 \gamma)$, i.e., when then Fabian algorithm is optimized for $S R, s(A S R)$ is close to $-2 \gamma$, close to 0 .

\subsection{Shamir and Fabian adapted for $A S R$}

Both algorithms presented in this section have a clear difference between the search and recommendation points. This fact is not automatically distinguished when we are evaluating their performance using for example a test bed. If we modify the algorithms we can achieve $A S R$ approximating well the optimal behavior reported by $S R$. A modification such as sampling one point out of two at the current recommendation, without using it in the algorithm can imply $s(A S R)=s(S R)$ arbitrarily close to -1 . 


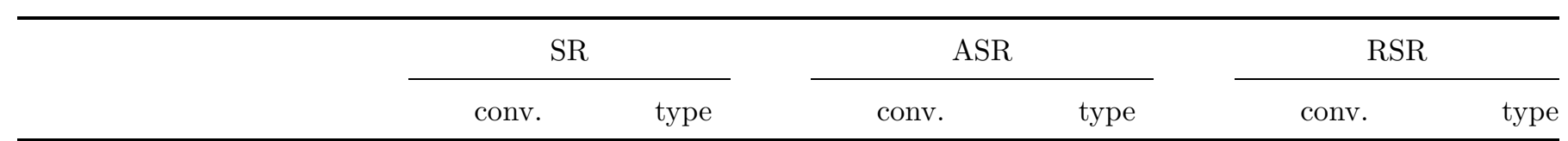

Evolutionary Algorithms

$\begin{array}{lll}\text { RS } & \mathbf{0} & \text { expect. }\end{array}$

$\mathrm{ES}+r \quad-\frac{1}{2} \quad$ high prob.

MES $+r \quad-\frac{1}{2} \quad$ high prob.

Stochastic Gradient

$\begin{array}{lll}\text { Shamir } & -1 & \text { expect. }\end{array}$

Shamir for ASR

expect.

Fabian $-(\mathbf{1}-\mathbf{e}) \quad$ a.s.

Fabian for ASR $\quad-(\mathbf{1}-\mathbf{e})$

a.s.

$\begin{array}{rr}-\frac{2}{\mathbf{d}} & \text { a.s. } \\ -\frac{2}{d} & \text { a.s. } \\ -\frac{1}{2} & \text { high prob. } \\ -\frac{1}{2}-\frac{2}{\mathbf{d}} & \text { high prob. }\end{array}$

a.s.

$\begin{array}{rr}-\frac{2}{\mathbf{d}} & \text { a.s. } \\ -\frac{2}{d} & \text { a.s. }\end{array}$

$-\frac{1}{2} \quad$ high prob.

$-\frac{1}{2}-\frac{2}{d} \quad$ high prob.

Table 1: Convergence rates for the regrets analysed on this paper. The "convergence" column refers to the convergence rate and the "type" column specifies the type of convergence: with high probability, in expectation, almost surely. The results in bold are proved and the others are conjectures, all of them presented in this paper. 


\section{Experiments}

We present experimental results for part of the algorithms theoretically analysed 6. We will analyse the convergence rate of this algorithms in terms of slope (see section 2.2 for the definition). As in the theoretical part, the function to optimize is the noisy sphere: $f(x)=\left\|x-x^{*}\right\|^{2}+\vartheta \mathcal{N}$ where $\vartheta=0.3$ and $\mathcal{N}$ is a standard gaussian distribution 7 . The dimension of the problem is 2 . The results in Fig. 11 correspond to the mean of 10 runs for each of the algorithms.

\begin{aligned} & \hline Algorithms Set of parameters \\ & \hline UHCMAES 21 $x_{\text {initial }}=1, \sigma_{\text {in }}=1 \\ &$ Shamir $\epsilon=0.3, \lambda=0.1, B=3 \\ &(1+1)$-ES \\ &$(1+1)$-ES resamp resamp $=2^{n} \\ &$ Fabian $\mathrm{s}=4 \alpha=1, \gamma=0.01 \\ &$\hline\end{aligned}

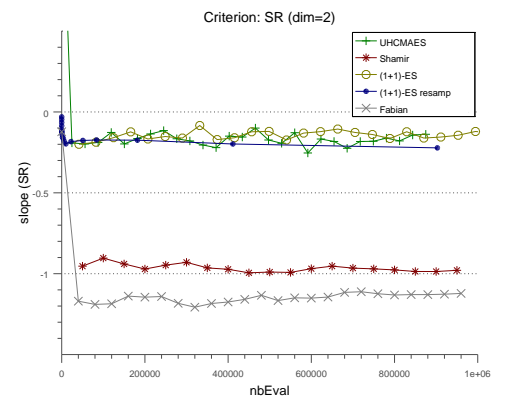

(a) Simple Regret

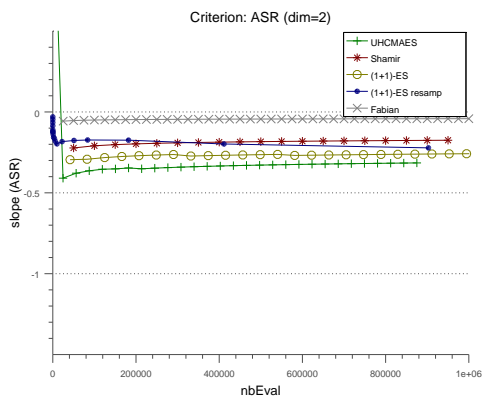

(b) Approximate Simple Regret

Figure 1: Figure 1a presents the Slope of Simple Regret for each algorithm on the first $\left(1 \cdot 10^{6}\right)$ function evaluations. Stochastic Gradient algorithms reach $s(S R)=-1$ while the evolutionary algorithms present $s(S R)=-0.2$. Figure1b presents the Slope of Approximate Simple Regret. Observe that the performance of the algorithms is inverted with regards to the figure 1a: now the Stochastic Gradient algorithms have the worse performance.

The results in figure (1a) show the comparison between algorithms with regards to the $S R$ criterion. The budget is limited to $\left(1 \cdot 10^{6}\right)$ function evaluations. We can see clearly the difference between the algorithm that are gradient-based and the $E S$. The algorithms Fabian and Shamir achieve $s(S R)=-1$ whereas the $E S$ presented cannot do better than $s(S R)=-0.25$. The figure (1b) shows that the use of $A S R$ changes completely the performance of the algorithms. In this case, the gradient-based algorithms are the ones with the worst perfor-

\footnotetext{
${ }^{6}$ In addition, the experimental results we include the algorithm $U H C M A E S$, as another example of an ES. For more information, see [21].

7 The choice $\vartheta=0.3$ is made only to illustrate in the experiments the effect of the regret choice in a reasonable time bugdet. The noise is weaker than in the case of a standard gaussian and the algorithms can deal with it faster. The optimum $x^{*}$ for the experiments of each algorithm is different, which does not affect the result since the regret compares the function value on the search/recommended points and on the optimum.
} 
mance. The results confirm the theoretical work (and the conjectures) presented in sections 3 and 4 .

\section{Conclusion}

In this paper we analyse the use of regrets to measure performance of algorithms in noisy optimization problems. We take into account the Simple Regret $(S R)$ and other two forms of regret used in practice to approximate the $S R$. We show that the convergence rates of the same algorithm over the same class of functions depend on the considered regret. Ultimately this leads to inconsistent results: algorithms are efficient for $S R$ and the opposite for an approximation of $S R$. Table 1 summarizes our results, detailing if they are proved or conjectured and what type of convergence is found.

Approximations of $S R$. The $A S R$ appears to be a poor approximation of $S R$. Two situations are exposed in this paper. First, algorithms with fast convergence for $S R$ can have a slow convergence for $A S R$ (Fabian and Shamir algorithm). However, this can be solved by modifying the algorithm in order to sample, sometimes, a recommended point. Second, an algorithm with slow convergence for $S R$ can have a fast convergence for $A S R$, and this is a serious issue. There is no simple "patch" to deal with this problem. Test beds using $A S R$ will underestimate algorithms which include random exploration. This is partially, but not totally, solved by $R S R$. We did not come up with a satisfactory regret definition, which would be consistent with $S R$ (at least showing similar convergence rates) and without drawbacks as those presented above. However, the difference (between $S R$ on the one hand, and $A S R / R S R$ on the other hand) decreases with the dimension for most algorithms.

Simple Regret. $S R$ is clearly a natural way to measure the quality of an approximated optimum output by an algorithm. The drawback of $S R$ is that it is not necessarily non-increasing, which is an issue for the concept of "stable first hitting time". Note that a particularly interesting result is the one of Evolution Strategies correctly tuned in terms of its resampling scheme. It appears, from theoretical and experimental results, that it reaches half the speed of classical noisy optimization algorithms in terms of $S R$, on the log-log scale. This corresponds to a squared computation time. 8

Further work. We have compared convergence rates through their slopes, but in some cases we have slopes for almost sure convergence, in other cases slope with high probability, and in others slope in expectation. There is room for refinement of the results. As for the approximations of $S R$, there might be other definitions of regret that approximate it in a better way. Evidently, the use of an approximation makes sense in the case where there are technical issues that do not allow the direct computation of $S R$.

\section{References}

[1] D. Arnold and H.-G. Beyer. Investigation of the $(\mu, \lambda)$-es in the presence of noise. In Proc. of the IEEE Conference on Evolutionary Computation

\footnotetext{
${ }^{8}$ This is not the case, however, for some ES with fast rates in noisy cases, such as mutatelarge-inherit-small 10.
} 
(CEC 2001), pages 332-339. IEEE, 2001.

[2] D. Arnold and H.-G. Beyer. Local performance of the $(1+1)$-es in a noisy environment. Evolutionary Computation, IEEE Transactions on, 6(1):30 -41 , feb 2002.

[3] D. V. Arnold and H.-G. Beyer. A general noise model and its effects on evolution strategy performance. IEEE Transactions on Evolutionary Computation, 10(4):380-391, 2006.

[4] S. Astete-Morales, M.-L. Cauwet, and O. Teytaud. Evolution Strategies with Additive Noise: A Convergence Rate Lower Bound. In Foundations of Genetic Algorithms, Foundations of Genetic Algorithms, page 9, Aberystwyth, United Kingdom, 2015.

[5] S. Astete-Morales, J. Liu, and O. Teytaud. log-log convergence for noisy optimization. In Proceedings of EA 2013, LLNCS, page accepted. Springer, 2013.

[6] A. Auger. Convergence results for $(1, \lambda)$-SA-ES using the theory of $\varphi$ irreducible Markov chains. Theoretical Computer Science, 334(1-3):35-69, 2005.

[7] A. Auger, D. Brockhoff, and N. Hansen. Benchmarking the (1, 4)-cma-es with mirrored sampling and sequential selection on the noisy bbob-2010 testbed. In GECCO (Companion), pages 1625-1632, 2010.

[8] A. Auger, D. Brockhoff, and N. Hansen. Investigating the impact of sequential selection in the $(1,4)$-cma-es on the noisy bbob-2010 testbed. In GECCO (Companion), pages 1611-1616, 2010.

[9] A. Auger, D. Brockhoff, and N. Hansen. Mirrored variants of the (1, 2)-cmaes compared on the noisy bbob-2010 testbed. In GECCO (Companion), pages $1575-1582,2010$.

[10] H.-G. Beyer. Mutate Large, But Inherit Small! On the Analysis of Rescaled Mutations in $(\tilde{1}, \tilde{\lambda})$-ES with Noisy Fitness Data. In Parallel Problem Solving from Nature, 5, Heidelberg, 1998. Springer. in print.

[11] H.-G. Beyer. The Theory of Evolution Strategies. Natural Computing Series. Springer, Heideberg, 2001.

[12] S. Bubeck, R. Munos, and G. Stoltz. Pure exploration in multi-armed bandits problems. In ALT, pages 23-37, 2009.

[13] M.-L. Cauwet. Noisy Optimization: Convergence with a Fixed Number of Resamplings. In EvoStar, Granada, Espagne, Apr. 2014.

[14] H. Chen. Lower rate of convergence for locating the maximum of a function. Annals of statistics, 16:1330-1334, Sept. 1988.

[15] D.-C. Dang and P. K. Lehre. Efficient optimisation of noisy fitness functions with population-based evolutionary algorithms. In Foundations of Genetic Algorithms 13, Lecture Notes in Computer Science, page Accepted. Springer-Verlag, Berlin Heidelberg, 2015. 
[16] P. Deheuvels. Strong bounds for multidimensional spacings. Z fur Wahrsch. Verw. Geb., 64:411-424, 1983.

[17] V. Dupač. O Kiefer-Wolfowitzově aproximační Methodě. Časopis pro pěstování matematiky, 082(1):47-75, 1957.

[18] V. Fabian. Stochastic Approximation of Minima with Improved Asymptotic Speed. Annals of Mathematical statistics, 38:191-200, 1967.

[19] S. Finck and H.-G. Beyer. Benchmarking spsa on bbob-2010 noisy function testbed. In GECCO (Companion), pages 1665-1672, 2010.

[20] S. Finck, H.-G. Beyer, and A. Melkozerov. Noisy optimization: a theoretical strategy comparison of es, egs, spsa \& if on the noisy sphere. In N. Krasnogor and P. L. Lanzi, editors, GECCO, pages 813-820. ACM, 2011.

[21] N. Hansen, S. Niederberger, L. Guzzella, and P. Koumoutsakos. A method for handling uncertainty in evolutionary optimization with an application to feedback control of combustion. IEEE Transactions on Evolutionary Computation, 13(1):180-197, 2009.

[22] N. Hansen and R. Ros. Benchmarking a weighted negative covariance matrix update on the bbob-2010 noisy testbed. In GECCO (Companion), pages 1681-1688, 2010.

[23] A. LaTorre, S. Muelas, and J. M. Pea. Benchmarking a mos-based algorithm on the bbob-2010 noisy function testbed. In GECCO (Companion), pages $1725-1730,2010$.

[24] L. Rastrigin. Convergence of random search method in extremal control of many parameter system. Automation and Remote Control, 24(11):1337, 1964.

[25] I. Rechenberg. Evolutionsstrategie. Friedrich Frommann Verlag (Günther Holzboog KG), Stuttgart, 1973.

[26] R. Ros. Black-box optimization benchmarking the ipop-cma-es on the noisy testbed: comparison to the bipop-cma-es. In GECCO (Companion), pages 1511-1518, 2010.

[27] R. Ros. Comparison of newuoa with different numbers of interpolation points on the bbob noisy testbed. In GECCO (Companion), pages 1495$1502,2010$.

[28] H.-P. Schwefel. Adaptive Mechanismen in der biologischen Evolution und ihr Einfluss auf die Evolutionsgeschwindigkeit. Interner Bericht der Arbeitsgruppe Bionik und Evolutionstechnik am Institut für Mess- und Regelungstechnik Re 215/3, Technische Universität Berlin, Juli 1974.

[29] O. Shamir. On the complexity of bandit and derivative-free stochastic convex optimization. In COLT 2013 - The 26th Annual Conference on Learning Theory, June 12-14, 2013, Princeton University, NJ, USA, pages 3-24, 2013. 
[30] J. Spall. Adaptive stochastic approximation by the simultaneous perturbation method. Automatic Control, IEEE Transactions on, 45(10):1839-1853, Oct 2000 .

[31] T.-D. Tran and G.-G. Jin. Benchmarking real-coded genetic algorithm on noisy black-box optimization testbed. In GECCO (Companion), pages 1739-1744, 2010.

\section{Supplementary material}

Theorem 1. With the framework above, for all $\beta>0$, the expected simple regret $\mathbb{E}\left(S R_{n}\right)$ is not $O\left(n^{-\beta}\right)$.

Lemma 1 (Logarithmic bounds on the quantile of the standard Gaussian variable). Let $Q(q)$ be $q$ quantile of the standard centered Gaussian, i.e. $\forall q \in$ $(0,1), P(\mathcal{N} \leq Q(q))=q$. Then $\forall \kappa \geq 1, \forall q \in(0,1)$,

$$
1-\sqrt{-\frac{2}{\kappa} \log (c(\kappa) q)} \leq Q(q) \leq 1-\sqrt{-2 \log (2 q)},
$$

where $c(\kappa)$ is a constant depending only on $\kappa$. In the following, we will denote by $X(q)$ (resp. $Y(q)$ ) the lower (resp.upper) bound on $Q: X(q)=$ $1-\sqrt{-\frac{2}{\kappa} \log (c(\kappa) q)}$ and $Y(q)=1-\sqrt{-2 \log (2 q)}$.

Proof. See http://arxiv.org/pdf/1202.6483v2.pdf.

Definition 1. We recall that we consider $n$ i.i.d search points $x_{1}, \ldots, x_{n}$. Let o and $\Omega$ be the standard Landau notations. Let $\epsilon: \mathbb{N} \backslash\{0\} \mapsto \mathbb{R}$ and $C: \mathbb{N} \backslash\{0\} \mapsto \mathbb{R}$ be two functions satisfying:

- $\forall n \in \mathbb{N} \backslash\{0\}, \epsilon(n)>0$ and $C(n)>0$,

- $\forall n \in \mathbb{N} \backslash\{0\}, \epsilon(n)=o(C(n))$,

- $\forall n \in \mathbb{N} \backslash\{0\}, \epsilon(n)=\Omega(1 / n)$ and $C(n)=\Omega(1 / n)$,

- $\forall n \in \mathbb{N} \backslash\{0\}, \epsilon(n)=o(1)$ and $C(n)=o(1)$.

Definition 2. With the previous definition of $\epsilon$ and $C$, consider the set $G$ defined by

$$
G:=\left\{i \in\{1, \ldots, n\} ;\left\|x_{i}-x^{*}\right\| \leq \sqrt{\epsilon(n)}\right\} .
$$

$G$ is the set of "good" search points, with simple regret better than $\epsilon(n)$. We denote by $N_{G}(n)$ the cardinality of $G$.

Definition 3. Similarly, consider the set B defined by

$$
B:=\left\{i \in\{1, \ldots, n\} ; \sqrt{\epsilon(n)}<\left\|x_{i}-x^{*}\right\| \leq \sqrt{C(n)}\right\} .
$$

$B$ is the set of "bad" search points, with simple regret bigger than $\epsilon(n)$, but still not that bad, since the simple regret does not exceed $C(n)$. We denote by $N_{B}(n)$ the cardinality of $B$. 
Lemma 2 (Linear numbers of good and bad points). There exist a constant $K_{d}>0$ such that, with probability at least $1 / 2$,

$$
N_{G}(n)<2 K_{d} n \sqrt{\epsilon(n)}^{d}
$$

and

$$
N_{B}(n) \geq 2 K_{d} n \sqrt{C(n)}^{d} .
$$

Proof. Proof of Eq. 6. Consider a search point $x_{i}$. The search points are drawn uniformly at random following the uniform distribution in $[0,1]^{d}$, then the probability $p$ that $x_{i} \in G$ is $p=\frac{\operatorname{Vol}\left(B_{d}\left(x^{*}, \sqrt{\epsilon(n)}\right)\right)}{\operatorname{Vol}\left([0,1]^{d}\right)}=K_{d} \sqrt{\epsilon(n)}^{d}$, where $V o l$ stands for 'volume' and $K_{d}$ is a constant depending on $d$ only. Therefore the number $N_{G}(n)$ of good points is the sum of $n$ Bernoulli random variables with parameter $K_{d} \sqrt{\epsilon(n)}^{d}$. The expectation is then $K_{d} n \sqrt{\epsilon(n)}^{d}$. By Markov inequality,

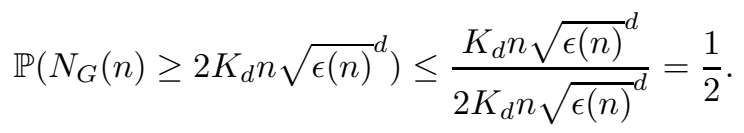

Similarly, $N_{B}(n)$ is a binomial random variable of parameters $n$ and $p=K_{d}\left(\sqrt{C(n)}^{d}-\sqrt{\epsilon(n)}^{d}\right)$. Then by Chebyshev's inequality, $\mathbb{P}\left(\left|N_{B}(n)-K_{d} n\left(\sqrt{C(n)}^{d}-\sqrt{\epsilon(n)}^{d}\right)\right| \leq \alpha\right) \geq 1 / 2$ by taking $\alpha=$

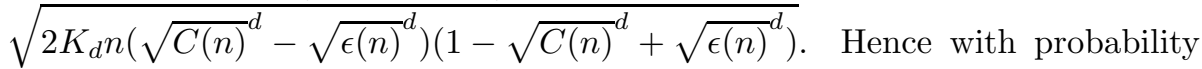
at least $1 / 2, N_{B}(n) \geq K_{d} n\left(\sqrt{C(n)}^{d}-\sqrt{\epsilon(n)}^{d}\right)+\alpha \geq 2 K_{d} n \sqrt{C(n)}^{d}$ since $\epsilon(n)=o(C(n))$.

We recall that $\forall i \in\{1, \ldots, n\}, y_{i}$ is the fitness value of search point $x_{i}$ : $y_{i}=\left\|x_{i}-x^{*}\right\|^{2}+\mathcal{N}_{i}$, where $\mathcal{N}_{i}$ is the realisation of a standard centered gaussian variable.

The following property gives a lower bound on the fitness values of the 'good' points, and an upper bound on the fitness values of the 'bad' points.

Proposition 1. With $X$ and $Y$ as defined in Lemma 1, and $C$ as defined in Definition [1, there exists some $c \in(0,1)$ such that, with probability at least $c$,

$$
\inf _{i \in G} y_{i} \geq X\left(1 / N_{G}(n)\right)
$$

and

$$
\inf _{i \in B} y_{i} \leq C(n)+Y\left(1 / N_{B}(n)\right) .
$$

Proof. Consider some Gaussian random variables independently identically distributed $\mathcal{N}_{1}, \ldots, \mathcal{N}_{N} . \forall i \in\{1, \ldots, N\}$, using notation of Lemma $1, \mathbb{P}\left(\mathcal{N}_{i} \leq\right.$ $Q(1 / N))=1 / N$, then $\mathbb{P}\left(\inf _{1 \leq i \leq N} \mathcal{N}_{i} \leq Q(1 / N)\right)=1-\mathbb{P}\left(\inf _{1 \leq i \leq N} \mathcal{N}_{i} \geq\right.$ $Q(1 / N)=1-(1-1 / N)^{N}$. The study of the function $x \mapsto 1-(1-1 / x)^{x}$ then shows that $\mathbb{P}\left(\inf _{1 \leq i \leq N} \mathcal{N}_{i} \leq Q(1 / N)\right) \in[1-\exp (-1), 3 / 4]$ (as soon as $N \geq 2$ ). 
Proof of Eq. 8 .

$$
\begin{aligned}
\mathbb{P}\left(\inf _{i \in G} y_{i} \geq X\left(1 / N_{G}(n)\right)\right)= & \mathbb{P}\left(\inf _{i \in G}\left\|x_{i}-x^{*}\right\|^{2}\right. \\
& \left.\quad+\mathcal{N}_{i} \geq X\left(1 / N_{G}(n)\right)\right) \\
\geq & \mathbb{P}\left(\inf _{i \in G}\left\|x_{i}-x^{*}\right\|^{2}\right. \\
& \left.\quad+\mathcal{N}_{i} \geq \epsilon(n)+X\left(1 / N_{G}(n)\right)\right) \\
\geq & \mathbb{P}\left(\inf _{i \in G} \mathcal{N}_{i} \geq X\left(1 / N_{G}(n)\right)\right) \\
\geq & 1-\mathbb{P}\left(\inf _{i \in G} \mathcal{N}_{i} \leq X\left(1 / N_{G}(n)\right)\right) \\
\geq & 1-\mathbb{P}\left(\inf _{i \in G} \mathcal{N}_{i} \leq Q\left(1 / N_{G}(n)\right)\right) \\
\geq & 1 / 4 .
\end{aligned}
$$

Proof of Eq. 9]

$$
\begin{aligned}
\mathbb{P}\left(\inf _{i \in B} y_{i} \leq C(n)+Y\left(1 / N_{B}(n)\right)\right)= & \mathbb{P}\left(\inf _{i \in G}\left\|x_{i}-x^{*}\right\|^{2}+\mathcal{N}_{i}\right. \\
& \left.\leq C(n)+Y\left(1 / N_{B}(n)\right)\right) \\
= & \mathbb{P}\left(\inf _{i \in B} \mathcal{N}_{i} \leq Y\left(1 / N_{B}(n)\right)\right) \\
\geq & \mathbb{P}\left(\inf _{i \in B} \mathcal{N}_{i} \leq Q\left(1 / N_{B}(n)\right)\right) \\
\geq & 1-\exp (-1)
\end{aligned}
$$

Hence, with probability at least 1/4, Eqs. 8 and 9 hold.

\section{Proof of Theorem 1;}

Proof. Let us assume that the expected simple regret has a slope $-\beta_{0}<-\beta$, for some $0<\beta<1$ : $\mathbb{E}\left(S R_{n}\right)=O\left(n^{-\beta_{0}}\right)$.

We define $\epsilon(n)=n^{-\beta}$. For some $0<k<1, \alpha=\beta / k$ and $C(n)=n^{-\alpha}$. $\epsilon$ and $C$ satisfy Definition 1 .

Lemma 2 and Proposition 1 implies that there is a $0<c<1$ such that with probability $c$, for $n$ sufficiently large,

- there are much more good points than bad points, i.e.

$$
N_{G}(n)=o\left(N_{B}(n)\right)
$$

thanks to Eq. 6] and [7.

- all good points have noisy fitness at least $X\left(1 / N_{G}(n)\right)=1$ $\sqrt{-\frac{2}{\kappa} \log \left(c(\kappa) / N_{G}(n)\right)}$

- at least one bad point has fitness at most $C(n)+Y\left(1 / N_{B}(n)\right)=n^{-\alpha}+$ $\left.1-\sqrt{-2 \log \left(2 / N_{B}(n)\right)}\right)$;

- therefore (by the two points above, using Eq. 10 and the fact that $n$ is big so that $n^{-\alpha}$ is negligeable), at least one bad point has a better noisy fitness than all the good points, and therefore is selected in Lines 7-9 of Alg. 1. 
This implies that

$$
\begin{aligned}
\mathbb{E}\left(S R_{n}\right) & =\mathbb{E}\left(\left\|x_{n}-x^{*}\right\|^{2}\right)=\mathbb{E}\left(\left\|x_{n}-x^{*}\right\|^{2} \mid x_{n} \in B\right) \mathbb{P}\left(x_{n} \in B\right) \\
& +\mathbb{E}\left(\left\|x_{n}-x^{*}\right\|^{2} \mid x_{n} \in G\right) \mathbb{P}\left(x_{n} \in G\right) \\
& \geq \mathbb{E}\left(\left\|x_{n}-x^{*}\right\|^{2} \mid x_{n} \in B\right) \mathbb{P}\left(x_{n} \in B\right) \\
& \geq \epsilon(n) \times c \\
& \geq c n^{-\beta} .
\end{aligned}
$$

We have a contradicion, hence $\beta_{0}>0$.

Theorem 2. Consider $0<\delta<1$. Consider an objective function with expectation $F(x)=\|x\|^{2}$, where $x \in \mathbb{R}^{d}$. Consider a $M E S+R$ as described previously (resampling number exponential in the number of iterations). Assume that:

(i) $\sigma_{n}$ and $\left\|\tilde{x}_{n}\right\|$ have the same order of magnitude:

$$
\left\|\tilde{x}_{n}\right\|=\Theta\left(\sigma_{n}\right)
$$

(ii) $\log -\log$ convergence occurs for the $S R$ :

$$
\frac{\log \left(\left\|\tilde{x}_{n}\right\|\right)}{\log (n)} \underset{n \rightarrow+\infty}{\longrightarrow}-\frac{1}{2} \text { with probability } 1-\delta
$$

Then, with probability at least $1-\delta, s(A S R)=-1 / 2-2 / d$.

Proof. We have $r_{n}=R \zeta^{n}$. In this proof we will index the recommendation and search points by the number of iterations instead of the number of evaluations. For $E S+r$, the recommendation point of the iteration $n$ is the corresponding center of the offspring distribution $\tilde{x}_{n}$. The step-size $\sigma_{n}$ corresponds to the standard deviation of the offspring distribution. The search points of iteration $n$ are the $\lambda$ offsprings produced on the iteration, denoted $\left\{x_{n}^{(i)}: i=1, \ldots, \lambda\right\}$.

We define $e_{n}$ the number of evaluations until the iteration $n$. From Algorithm 2 we have $e_{n}=\lambda \sum_{i=1}^{n} r_{i}$ for an ES with resampling $r$. By hypothesis (Eq. 4) we know the convergence of the sequence $S_{n}$ defined as the logarithm of the recommendation points, indexed by the number evaluations, divided by the logarithm the number of evaluations. Therefore, the sequence $\mathcal{S}_{n}=\log \left(\left\|x_{n}\right\|\right) / \log \left(e_{n}\right)$ is a subsequence of $S_{n}$, hence convergent to the same limit, with the same probability. The relation on Eq. 3 also remains the same after the index modification. From these facts we can inmediatly conclude that $\exists n_{0}$ such that

$$
\sigma_{n}=\Theta\left(e_{n}^{-1 / 4}\right) \text { for } n \geq n_{0} .
$$

For the $M E S+r$ algorithm, the $A S R_{n}$ is defined as:

$$
A S R_{n}=\min _{m \leq n} \min _{1 \leq i \leq \lambda+r_{m}}\left\|x_{m}^{\prime(i)}\right\|^{2}
$$

where $\left\{x_{n}^{(i)}: i=1, \ldots, \lambda+r_{m}\right\}$ considers all the search points at iteration $n$. Both the "real" and the "fake" ones. We will find a bound for $A S R_{n}$, which will lead us to the convergence rate of the $A S R$ for the $M E S+r$ algorithm. 
Let $p_{x}$ be the probability density at point $x$ of the offsprings in iteration $n$. Therefore it is the probability density of a Gaussian centered at $\tilde{x}_{n}$ and with variance $\sigma_{n}^{2}$. At the origin:

$$
\begin{aligned}
p_{0} & =\frac{1}{(2 \pi)^{d / 2} \sigma_{n}^{d}} \exp \left\{-\frac{1}{2} \frac{\left(-\tilde{x}_{n}\right)^{T}\left(-\tilde{x}_{n}\right)}{\sigma_{n}^{2}}\right\} \\
& =\Theta\left(\sigma_{n}^{-d}\right) \text { by Eq. } 3 \\
& =\Theta\left(e_{n}^{d / 4}\right) \text { by Eq. } 13
\end{aligned}
$$

Now, at iteration $n$, the probability to have at least one offspring with norm less than $\epsilon>0$ is

$$
\begin{aligned}
\mathbb{P}\left(\exists i:\left\|{x^{\prime}}_{n}^{(i)}\right\| \leq \epsilon\right) \quad \leq & \left(\lambda+r_{n}\right) \mathbb{P}\left(\left\|x_{n}^{\prime(i)}\right\| \leq \epsilon\right) \\
& \leq\left(\lambda+r_{n}\right) \int_{\|x\| \leq \epsilon} d p_{x}
\end{aligned}
$$

By Eq. 15,

$$
\mathbb{P}\left(\exists i \mid\left\|x_{n}^{\prime(i)}\right\| \leq \epsilon\right)=\Theta\left(r_{n} \cdot e_{n}^{d / 4} \epsilon^{d}\right)=\Theta(1)
$$

if $\epsilon=\Theta\left(e_{n}^{-1 / 4} \cdot r_{n}^{-1 / d}\right)$. Therefore we obtain:

$$
\begin{aligned}
A S R_{n} & \leq \min _{1 \leq i \leq \lambda+r_{n}}\left\|x_{n}^{(i)}\right\|^{2} \\
& \leq \epsilon^{2} \\
& =O\left(e_{n}^{-1 / 2} \cdot r_{n}^{-2 / d}\right) \\
& =O\left(e_{n}^{-1 / 2-2 / d}\right)
\end{aligned}
$$

Since $e_{n}=(1+\lambda) \sum_{i=1}^{n} r_{i}=(1+\lambda) \cdot R \sum_{i=1}^{n} \zeta^{i}=\Theta\left(r_{n}\right)$ Hence we have the result, $s(A S R) \leq-1 / 2-2 / d$.

Theorem 5 (ASR of Fabian algorithm). Let $F$ be a $\lambda$-convex and $\mu$-smooth function corrupted by an additive noise with upper bounded density and with optimum randomly drawn according to a distribution with upper bounded density. Then, a.s.,

$$
s(A S R)=-\min (2 \beta, 2 \gamma) .
$$

Proof. The upper bounded density is used (Theorem 3 section 4.1)) for ensuring that no recommendation is never exactly equal to the optimum.

Using the algorithm notations,

$$
A S R_{n}=\min _{n, i, j} F\left(x^{(i, j)+}(n)\right)-F\left(x^{*}\right)
$$

(or $\min _{n, i, j} F\left(x^{(i, j)-}(n)\right)-F\left(x^{*}\right)$, which does not affect the proof), where $x^{(i, j)+}(n)$ is the $n^{\text {th }}$ search point. Noting that since there is $s \times d$ evaluations per iterations, $\tilde{x}$ is updated only every $s \times d$ evaluations, so we have $\tilde{x}_{n}=\tilde{x}_{n+1}=\cdots=\tilde{x}_{n+s \times d-1}$, therefore $x^{(i, j)+}(n)=\tilde{x}_{\lfloor n / s \times d\rfloor}+c_{n} u_{j} e_{i}$. By the convexity of $F$, the fact that the gradient of $F$ in $x^{*}$ is 0 ,

$$
F\left(x^{(i, j)+}(n)\right)-F\left(x^{*}\right) \geq \frac{\lambda}{2}\left\|\left(\tilde{x}_{\lfloor n / s \times d\rfloor}-x^{*}\right)+\left(c_{n} u_{j} e_{i}\right)\right\|^{2}
$$


Similarly, by using the $\mu$-smoothness of $F$,

$$
F\left(x^{(i, j)+}\right)-F\left(x^{*}\right) \leq \frac{\mu}{2}\left\|\left(\tilde{x}_{\lfloor n / s \times d\rfloor}-x^{*}\right)+\left(c_{n} u_{j} e_{i}\right)\right\|^{2}
$$

Then

$$
F\left(x^{(i, j)+}(n)\right)-F\left(x^{*}\right)=\Theta\left(\left\|\left(\tilde{x}_{\lfloor n / s \times d\rfloor}-x^{*}\right)+\left(c_{n} u_{j} e_{i}\right)\right\|^{2}\right)
$$

If $\beta>\gamma$, then the main term is the last one and we get a rate $-2 \gamma$. If $\beta \leq \gamma$, then the main term is the first one and we get a rate $-2 \beta$. 